\title{
Análise de equações preditivas da gordura corporal em jovens atletas de "taekwondo"
}

CDD. 20.ed. 796.022

796.84

\author{
Pedro Vieira Sarmet MOREIRA* \\ Analiza Mónica SILVA ${ }^{* * *}$ \\ Luciano Fernandes CROZARA ${ }^{* * *}$ \\ António Prieto VELOSO* \\ Filomena VIEIRA*
}

*Faculdade de Motricidade Humana, Universidade Técnica de Lisboa - Portugal. **Instituto de Pesquisa Médica, Universidade de Oklahoma - EUA. ${ }^{* * *}$ Universidade Estadual Paulista.

\section{Resumo}

Devido à falta de métodos acessiveis válidos para mensurar o percentual de gordura corporal (\%G) de taekwondistas adolescentes (TKDA), objetivou-se analisar seis equações antropométricas de predição do $\%$ G, em cinco TKDA $(12,23$ anos $\pm 1,60)$, utilizando como método de referência a Densitometria Radiológica de Dupla Energia (DEXA). Os \%G estimados pelas equações foram comparados pelo teste t-student, regressão linear e Bland e Altman (BCAA) com os obtidos por DEXA. Apenas a equação de SLAUGHTER et

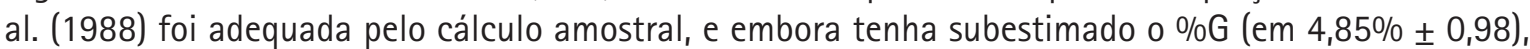
esta apresentou alta correlação $\left(R=0,935 ; R^{2}=0,874 \quad E P E=1,01\right)$ e baixa amplitude nos limites de concordância a 95\% (3,84\%) pelo B\&tA em comparação com a DEXA. Portanto, esta equação mostrou-se adequada para a predição do $\% \mathrm{G}$ em TKDA, desde que seja corrigida pela equação de ajuste [\%G (DEXA) $=1,64+1,24 \cdot \% \mathrm{G}(\mathrm{Eq} 4)]$ gerada pela regressão linear.

UNITERMOS: Densitometria; Composição corporal; Dobras-cutâneas.

\section{Introdução}

O "Taekwondo" (TKD) é um desporto de combate, o que implica aos atletas competir em categorias de peso bem definidas. Isto justifica a importância da avaliação da composição corporal destes atletas, pois se supóe que o aumento em sua massa corporal devido a um acréscimo dos níveis de gordura corporal pode levar a um decréscimo no desempenho. Confirmando esta hipótese, Heller, Peric, Douhá, Kohlíková, Melichna e Nováková (1998) demonstraram haver correlação inversa significativa do percentual de gordura $(\% \mathrm{G})$ com a classificação de atletas de elite de TKD no "ranking" internacional.

A necessidade de métodos rápidos e de baixo custo para mensurar a composição corporal de atletas explica a popularidade da antropometria (WANG, Thorton, Kolesnik \& Pierson, 2000), mas a falta de métodos válidos e de referência padrão em atletas jovens para tal objetivo, não permite uma correta orientação ao nível da composição corporal ideal a se alcançar e nem que a antropometria possa ser realizada de forma segura quanto à credibilidade e precisão dos resultados. Os erros de precisão associados às equaçóes antropométricas podem ocasionar tanto a superestimação como a subestimação do \%G.

A superestimação do \%G de atletas, para a elaboração do treino ou de metas competitivas, pode aumentar o risco do desenvolvimento de transtornos alimentares e problemas relacionados com o déficit nutricional de energia, proteínas e micronutrientes (Petrie, Stover \& Horswill, 2004). A utilização de dietas inadequadas pode causar prejuízos no desempenho e saúde de atletas jovens, tanto a curto como em longo prazo (Fleming \& Costarelli, 2007), tais como, distúrbios no crescimento, aumento do risco de fraturas (COTUGNA, VickeRY \& McBeE, 2005), osteopenia, anemia e síndromes relacionadas com deficiências em vitaminas, minerais, ácidos graxos essenciais e aminoácidos, incluindo a diminuição do metabolismo basal e da síntese protéica (Rogol, Clark \& RoemMICH, 2000), com implicações no desenvolvimento 
muscular e provável diminuição do condicionamento físico e da produção de potência. Além disto, o estudo de Timpson, Sayers, Davey-Smith e Tobias (2009) demonstrou haver relação causal dentre os valores de gordura corporal e o conteúdo mineral ósseo.

Por outro lado, a subestimação da massa gorda de taekwondistas pode diminuir suas probabilidades de sucesso competitivo, devido ao consequente erro no planejamento do volume e dispêndio energético de treino, ingestão calórica e categorias de peso em que cada atleta deve se enquadrar para alcançar metas de composição corporal, pois, dentro dos limites saudáveis, se almeja baixo \%G. Isto fornece uma melhor relação força/peso corporal, que por sua vez realça a produção de potência relativa, um elemento importante para a aceleração da massa corporal tão rápido quanto possível (TAAfFe \& Pieter, 1990).

A Densitometria Radiológica de Dupla Energia (DEXA) pode ser considerada como um método de referência para a avaliação da composição corporal (Massa Magra e Massa Livre de Gordura), devido à sua precisão de estimativa ao nível individual (PARKer, ReILly, Slater, Wells \& Pitsiladis, 2003; Rodríguez, Moreno, Blay, Blay, Fleta, Sarría \& Bueno, 2005). Existem estudos comparativos que confrontam a antropometria com métodos padrão em adultos (GobBo, Cyrino, Petroski,

\section{Método}

\section{Amostra}

Utilizou-se para a seleção da amostra os seguintes critérios: a) Ser do gênero masculino e possuir idade menor ou igual a 14 anos; b) Ter sido medalhado no campeonato Português de Cadetes ou em competição internacional em 2008; c) Não ser de qualquer categoria de Peso superior a $53 \mathrm{~kg}$.

Dos cinco atletas selecionados, dois foram vencedores do Campeonato Nacional de Portugal (CNP), de cadetes (sub 14 anos), em 2008 e outros dois em 2009. Além disto, um atleta foi medalhado em segundo lugar e outro em terceiro lugar no CNP de cadetes de 2008. Neste mesmo ano, um participante foi medalhado em campeonato internacional aberto (Open do Rio Grande do Sul) oficializado pela World Taekwondo Federation (WTF). No ano de 2009, dois atletas da amostra foram medalhados no torneio internacional (Open da Espanha, 2009) classe A pela WTF, e participaram do Campeonato Europeu de Cadetes 2009, sendo que um destes conquistou o quinto lugar nesta competição.
Cardos, Carvalho, Romanzini \& Avelar, 2008; Paccini, Arsa \& Glaner, 2008; Silva, Fields, Quttério \& Sardinha, 2009), idosos (Rech, Lima, Cordeiro, Petroski \& Vasconcelos, 2010) e alguns em crianças e adolescentes não atletas (CAMPANOZZI, DABBAS, RUIZ, Ricour \& GOUlet, 2008; Loftin, Nichols, GoING, SOTHERn, SCHMitZ, Ring \& STEVEns, 2007; RodRíGUEZ et al., 2005). No entanto, encontrar pesquisas nesta temática realizadas com jovens atletas é uma tarefa difícil. Especificamente à modalidade "taekwondo", nenhuma pesquisa comparativa sobre métodos de estimação do $\% \mathrm{G}$ foi encontrada. O mesmo pode ser dito quanto à disponibilidade de padrões de $\% \mathrm{G}$ para taekwondistas infantis e adolescentes, pois embora tais padrōes de lutadores adultos já tenham sido descritos (OlDS \& KANG, 2000; Pieter \& BerCADES, 2009), estudos nesta modalidade feitos com atletas tão jovens são escassos.

Esta escassez na literatura específica faz com que qualquer meta de composição corporal a alcançar em taekwondistas infanto-adolescentes, bem como a escolha de métodos credíveis para tal mensuração em campo, sejam meramente especulativas. Desta forma, o objetivo deste estudo foi analisar diferentes equações baseadas em dobras cutâneas na estimação do $\% \mathrm{G}$ em jovens competidores de TKD, utilizando como método referência a DEXA.
Todos os voluntários treinavam de três a seis horas semanais, por mais de dois anos. Os participantes assinaram o termo de consentimento de livre esclarecimento aprovado pelo comitê de ética da Faculdade de Motricidade Humana (FMH-UTL) (08/09/2009). Cada atleta realizou todos os testes em um único dia, na FMH-UTL, nos laboratórios de Exercício e Saúde e de Biomecânica e Morfologia Funcional.

\section{Antropometria}

A massa corporal foi medida em uma Balança - Seca, Vogel \& Halke (Germany) modelo 761 7019009, com aproximação de $1 \mathrm{~kg}$. A estatura foi obtida com o estadiômetro - Siber Hegner Machines, SA. Zurich (precisão $1 \mathrm{~mm}$ ); e as pregas adiposas foram medidas com um adipômetro Slimguide (precisão $1 \mathrm{~mm}$ ). As dobras cutâneas avaliadas foram: biceptal, triceptal, subscapular, supra-ilíaca, da panturrilha e da coxa. Todas as medidas foram coletadas em duplicata por um único avaliador, creditado pelo 
ISAK (International Society for the Advancement in Anthropometry) com o nível 4 . O erro técnico de medida foi aceitável, pois seguiu o padrão da ISAK ao não exceder a $7,5 \%$ para as pregas adiposas e a $1,5 \%$ para todas as outras medidas.

\section{Densitometriaradiológicadeduplaenergia(DEXA)}

A DEXA (QDR-4500, Hologic, Watham, USA, versão 8.21) foi utilizada neste estudo como método de referência para o cálculo da massa gorda. Este equipamento mede a atenuação dos raios-x emitidos com frequências entre os 70 e $140 \mathrm{kV}$ sincronizados com a frequência de linha para cada pixel da imagem recolhida através do "scan". Foram seguidas as instruçōes descritas no manual do fabricante do equipamento, de acordo com as quais se colocou ao longo de cada indivíduo um "phantom" constituído por três campos de material acrílico e três campos de alumínio com diferentes espessuras e com propriedades absortivas conhecidas, para servir como referência externa para a análise da diferente composição dos tecidos. O "software" do sistema fornece a indicação da massa de gordura, tecido mole isento de gordura e conteúdo mineral, quer para o corpo inteiro, como para regiōes específicas (cabeça, tronco, membros superiores e inferiores). O mesmo técnico que posicionou os sujeitos, realizou os "scans" e efetuou as análises de acordo com o manual do operador. Todos os indivíduos foram avaliados após 12 horas em jejum. Com base em duas avaliações realizadas em dois dias consecutivos em 10 sujeitos, o erro técnico de medição na avaliação do \%G é de 2,9\%.

\section{Maturação óssea}

A avaliação da maturação óssea fez-se por meio de um exame radiológico da mão e pulso esquerdo, utilizando aparelho de Rx portátil modelo Ascot 110, chassis modelo Kodak Min-R 2 e películas Min- $\mathrm{R}$ também da Kodak. A idade óssea foi determinada em acordo ao método de Tanner-Whitehouse (TW3) (Tanner, Healy, Goldstein \& Cameron, 2001).

\section{Equações antropométricas}

Foram utilizadas equaçōes (Eq) específicas para crianças e adolescentes do sexo masculino, como mostradas na TABELA 1. Encontrou-se seis equações cuja as amostras utilizadas em seus desenvolvimentos abrangiam a faixa-etária completa (9-14 anos) da atual amostra. As equaçóes 1 e 5 , foram realizadas para o cálculo da Densidade Corporal (D), para posteriormente ser convertida em $\% \mathrm{G}$, pela equação "WD", enquanto as equações restantes resultavam diretamente no valor predito de \%G.

TABELA 1 - Equações para o cálculo do \%G e da densidade corporal por meio de pregas cutâneas.

\begin{tabular}{|c|c|c|c|}
\hline IE & Autor & ID (anos) & Equação \\
\hline \multirow[t]{2}{*}{$1^{*}$} & PARIZKOVA (1961) & $9-13$ & $\mathrm{D}=1,108-0,027\left(\log _{10} \mathrm{TR}\right)-0,0388\left(\log _{10} \mathrm{SE}\right)$ \\
\hline & & $13-16$ & $\mathrm{D}=1,130-0,055\left(\log _{10} \mathrm{TR}\right)-0,026\left(\log _{10} \mathrm{SE}\right)$ \\
\hline 2 & BOILEAU, LOHMAN e SLAUGHTER (1985) & $8-28$ & $\% \mathrm{G}=1,35(\mathrm{TR}+\mathrm{SE})-0,012(\mathrm{TR}+\mathrm{SE})^{2}-4,4$ \\
\hline 3 & LOHMAN (1986) & $6-17$ & $\% \mathrm{G}=1,35(\mathrm{TR}+\mathrm{SE})-0,012(\mathrm{TR}+\mathrm{SE})^{2}-\mathrm{C}$ \\
\hline 4 & SLAUGHTER et al. (1988) & $8-29$ & $\% \mathrm{G}=0,735(\mathrm{TR}+\mathrm{PA})+1$ \\
\hline $5^{*}$ & JoHnSTON et al. (1988) & $8-14$ & $\mathrm{D}=1,166-0,07\left(\log _{10} \Sigma 4\right.$ dobras $)$ \\
\hline 6 & MukHERJeE e Roche (1984) & $6-18$ & $\% \mathrm{G}=12,66-0,85$ (idade) $+1,10(\mathrm{TR})+0,53(\mathrm{PA})$ \\
\hline WD & Westrate e DeURENBERG (1989) & $2-18$ & $\% G=(\{562-4,2[\mathrm{ID}-2]\} / \mathrm{D})-\{525-4,7[\mathrm{ID}-2]\}$ \\
\hline
\end{tabular}

\section{Análise estatística}

As análises estatísticas foram feitas com o "Statistical Package for the Social Sciences (SPSS)" "software" 17.0 (SPSS Inc., Chicago, IL, USA). Para o cálculo de potência dos testes, "effect size" e tamanho amostral foi utilizado o G*Power "software” 3.1 .3 (Universitat Kiel, Germany).
Após feita a estatística descritiva, as distribuições dos valores de $\% \mathrm{G}$ por cada equação e por DEXA foram testadas em normalidade, usando os testes de Kolmogorov-Smirnov e Shapiro-Wilk. A normalidade das diferenças entre os valores de \%G obtidos por DEXA e por cada equação antropométrica foi também testada. Apenas a distribuição dos dados das diferenças da equação 6 não obteve normalidade.
$\mathrm{IE}=$ Identificação da Equação; $\mathrm{D}=$ densidade Corporal $(\mathrm{kg} / \mathrm{l})$;

$\% \mathrm{G}=$ percentual de gordura corporal;

* = A D obtida pelas equações 1 e 5 , foi transformada em $\% \mathrm{G}$ por meio da equação WD; Log = Logarítmica $\Sigma 4$ dobras $(\mathrm{mm})$ : bíceps + tríceps + subscapular + suprailiaca $(\mathrm{mm})$;

TR = dobra triceptal (mm);

$\mathrm{PA}=$ dobra da panturrilha (mm);

SE = Subscapular; $\mathrm{C}=$ Constante por idade $(4,4 ; 4,7 ; 5,0 ; 5,4$ e 5,7 para $10,11,12,13$ e 14 anos, respectivamente); ID = Idade em anos. 
O teste t-student e o de Wilcoxon foram utilizados para comparação de médias das equações com e sem distribuição normal em todas as variáveis, respectivamente; as correlaçôes de Pearson e de Spearman foram utilizadas para determinar o poder explicativo e o erro padrão de estimação das equações com e sem distribuição normal em todas as variáveis, respectivamente. Utilizando cinco indivíduos, este estudo apresentou um poder estatístico de $80 \%$ para detectar um coeficiente de correlação maior que 0,90 , nos testes de Pearson e Spearman e para detectar um "effect size" de 1,7 ou maior por meio do teste t-Student, e de 1,75 ou maior para o teste de Wilcoxon. Após o cálculo amostral, o teste de BLAND e AltMAN (1986) foi realizado apenas com a equação que possuiu um poder estatístico igual ou superior a $80 \%$ na determinação da variabilidade dos valores obtidos por DEXA, para verificar a concordância entre métodos, incluindo os limites de concordância a 95\%. A correlação entre a média dos valores de $\% \mathrm{G}$ estimada pelo método de referência e por antropometria com a diferença dentre ambos foi utilizada como um indicador de tendência (i.e., se a diferença dentre métodos variou de acordo com o incremento dos níveis de gordura corporal). Para todos os procedimentos, considerouse a significância de $\mathrm{p}<0,05$.

\section{Resultados}

Os dados descritivos básicos de cada atleta e os valores de \%G obtidos por DEXA, e pelas equaçôes antropométricas 1 a 6 estão mostrados na TABELA
2, além disto, os valores de massa magra, massa gorda e conteúdo mineral ósseo obtidos por DEXA, estão também disponíveis nesta TABELA 2.

TABELA 2 - Dados descritivos da amostra, e \%G obtido por DEXA e pelas equações.

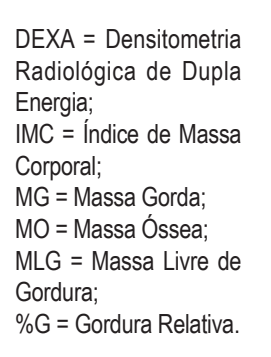

\begin{tabular}{|c|c|c|c|c|c|c|c|}
\hline \multirow{2}{*}{ Variáveis } & \multicolumn{5}{|c|}{ Atletas } & \multirow{2}{*}{ Média } & \multirow{2}{*}{ DP } \\
\hline & 1 & 2 & 3 & 4 & 5 & & \\
\hline Idade biológica & 12,05 & 9,12 & 13,32 & 14,02 & 11,75 & 12,05 & 1,88 \\
\hline Idade cronológica & 11,5 & 10 & 14,25 & 13 & 12,42 & 12,23 & 1,60 \\
\hline Massa & 37 & 25,2 & 40,6 & 51,5 & 36,8 & 38,22 & 9,42 \\
\hline Estatura $(\mathrm{cm})$ & 149,8 & 137,5 & 163 & 169 & 147,4 & 153,3 & 12,6 \\
\hline IMC $\left(\mathrm{kg} / \mathrm{m}^{2}\right)$ & 16,49 & 13,33 & 15,28 & 18,03 & 16,94 & 16,01 & 1,8 \\
\hline MG (DEXA) (kg) & 6,72 & 4,20 & 8,52 & 7,6 & 7,21 & 6,85 & 1,62 \\
\hline MO (DEXA) (kg) & 1,087 & 1,082 & 1,330 & 1,706 & 1,480 & 1,337 & 0,267 \\
\hline MLG (DEXA) (kg) & 39,13 & 20,81 & 31,52 & 43,22 & 29,33 & 32,80 & 8,75 \\
\hline$\% G(D E X A)$ & 18,2 & 16,8 & 21,3 & 15 & 19,7 & 18,2 & 2,45 \\
\hline \% G (Equação 1) & 15,92 & 12,00 & 14,52 & 15,31 & 18,16 & 15,18 & 2,23 \\
\hline \% G (Equação 2) & 13,15 & 10,07 & 12,15 & 11,12 & 16,01 & 12,50 & 2,27 \\
\hline \% G (Equação 3) & 12,85 & 10,07 & 10,85 & 10,12 & 15,41 & 11,86 & 2,28 \\
\hline \% G (Equação 4) & 13,50 & 11,66 & 14,97 & 11,29 & 15,33 & 13,35 & 1,85 \\
\hline \% G (Equação 5) & 9,77 & 6,12 & 11,85 & 10,65 & 12,58 & 10,20 & 2,52 \\
\hline \% G (Equação 6) & 17,03 & 15,84 & 15,75 & 13,31 & 18,28 & 16,24 & 2,17 \\
\hline
\end{tabular}

O resultado das comparações das médias, dentre os valores percentuais de gordura obtidos pelas fórmulas antropométricas (1 a 6) e aqueles resultantes da análise por DEXA estão mostrados na TABELA 3. 
TABELA 3 - Diferenças dos \%G obtidos pelas equações antropométricas e por DEXA.

\begin{tabular}{lcccccc}
\hline & T (ou Z) & $\mathbf{R}$ & $\mathbf{r}^{\mathbf{2}}$ & $\mathbf{E P E}$ & $\mathbf{E C}(\mathbf{D P})$ & ET (\%) \\
\hline Equação 1 & $2,42^{\mathrm{a}}$ & 0,295 & 0,087 & 2,71 & $3,02(2,79)$ & 3,91 \\
Equação 2 & $5,59^{\mathrm{a}}$ & 0,537 & 0,289 & 2,39 & $5,70(2,28)$ & 6,05 \\
Equação 3 & $5,74^{\mathrm{a}}$ & 0,459 & 0,210 & 2,52 & $6,34(2,47)$ & 6,71 \\
Equação 4 & $11,09^{\mathrm{a}}$ & $0,935^{\mathrm{b}}$ & 0,874 & 1,01 & $4,85(0,98)$ & 4,93 \\
Equação 5 & $7,37^{\mathrm{a}}$ & 0,524 & 0,274 & 2,41 & $8,00(2,43)$ & 8,29 \\
Equação 6 $(\mathrm{Z})$ & $-2,02^{\mathrm{a}}$ & 0,400 & 0,16 & - & $1,96(2,06)$ & 2,69 \\
\hline
\end{tabular}

O percentual de gordura médio obtido pelo método de referência foi $18,2 \%( \pm 2,45)$. Enquanto que das formulas antropométricas, a equação 5 foi a que obteve os menores valores $(10,20 \% \pm 2,52)$, sendo portanto aquela que mais subestimou o percentual de massa gorda, em $8 \%( \pm 2,43)$, com $\mathrm{p}<$ 0,001; seguida pelas equações 3, 2, 4, 1 e 6 .

A análise de regressão indicou que a equação com maior poder explicativo foi a equação 4 , cuja o coeficientes de determinação $\left(R^{2}\right)$ foi $0,874(p=$ $0,01)$. Devido ao $\mathrm{R}$ encontrado ter sido superior a $0,90(0,935)$, esta foi a única equação cuja o tamanho amostral se mostrou adequado para a regressão linear, que resultou na seguinte equação de ajuste:
$\% \mathrm{G}(\mathrm{DEXA})=1,64+1,24 \cdot \% \mathrm{G}(\mathrm{Eq} 4)$

Portanto, os resultados de $\% \mathrm{G}$ preditos pela a equação 4 foram os únicos a terem concordância com os \%G obtidos por DEXA testados através da plotagem de Bland e AltMAn (1986) (FIGURA 1).

A equação 4 resultou em uma amplitude dos limites de concordância igual a 3,84\% que pode ser considerada adequada. É importante observar que não se obteve correlação significativa dentre a diferença do \%G obtido pela equação e por DEXA com a média dentre métodos. Isto indicou não haver para a Equação 4, associação dentre a magnitude dos erros e os valores de $\% \mathrm{G}$ obtidos pelos atletas, o que significa que este método não apresenta um enviesamento.
${ }^{\mathrm{a}}=$ Os resultados determinados pelo método de referência (DEXA) e os estimados pela equação preditiva diferem estatisticamente. $(p<0,05)$;

${ }^{b}=$ Houve correlação significativa entre os valores estimados pela equação preditiva e 0 método padrão;

$Z$ = Significa que nesta equação se realizou 0 teste de Wilcoxon para a comparação de médias, em alternativa ao teste t-student;

EPE = Erro Padrão de Estimativa;

$\mathrm{EC}=$ Erro Constante;

$\mathrm{ET}=$ Erro Total.

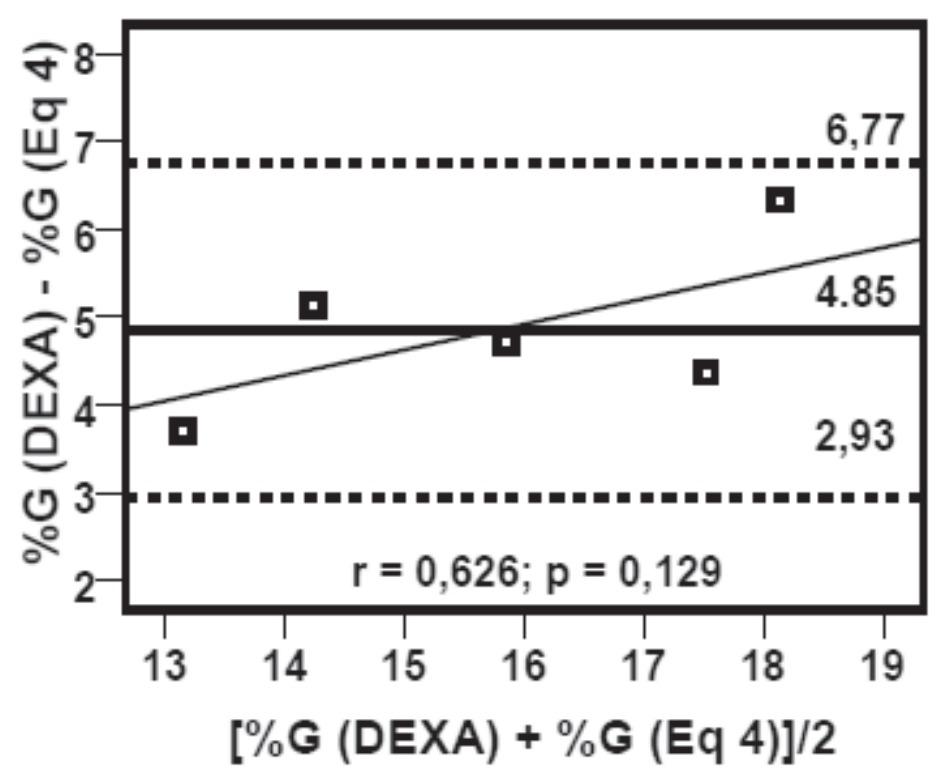

A linha sólida do meio representa a diferença média dentre $0 \% \mathrm{ob}$ tido por DEXA o o obtido pela Equação 4; As linhas tracejadas superiores e inferiores representam $\pm 1,96 \mathrm{DP}$ da média (i.e. $95 \%$ dos limites de concordância); A linha de tendência representa a associação dentre as diferenças de métodos ( $\%$ G pela Equação 4 menos o valor por DEXA) e a média de ambos os métodos em estimar $0 \% \mathrm{G}$.

FIGURA 1 - Análise de BLAND e ALtMAN (1986) para a concordância dentre métodos em relação ao \%G. 


\section{Discussão}

Para analisar a concordância de seis equações antropométricas, na predição do percentual de gordura corporal, em crianças e adolescentes $(12,23$ anos $\pm 1,60$ ) do gênero masculino, competidores de "taekwondo", utilizando como método de referência a Densitometria Radiológica de Dupla Energia, cinco taekwondistas adolescentes participaram deste estudo.

O percentual médio de gordura obtido no atual estudo foi semelhante ao encontrado no estudo original de Slaughter, Lohman, BoIleau, Horswill, Stillman, Van Loan e Bemben (1988), que originou na equação 4. Nesta pesquisa, os voluntários obtiveram valores relativos de $19 \pm 8,1 \% ; 17,3 \pm 7,3 \%$ e $14 \pm$ $6,2 \%$ de gordura, respectivamente. O mesmo ocorreu ao comparar nossos dados com aqueles do estudo de Quitério, Silva, Carnero e Sardinha (2007), que analisaram 70 atletas portugueses infantis e adolescentes (14,6 $\pm 2,1$ anos), do sexo masculino, praticantes de diferentes modalidades. Estes autores obtiveram por DEXA, uma média de 18,2 ( \pm 6,8 \%), mesmo com volumes de treino semanal superiores ao atual $(11,1 \pm$ 8,8 horas vs 3-6 horas, respectivamente). Apesar disto, os atletas da atual pesquisa demonstraram uma maior homogeneidade que ambos os estudos supracitados, o que pode ser explicado pelo fato de todos os nossos voluntários serem atletas e praticantes da mesma modalidade, além de treinarem o mesmo volume semanal, com as mesmas intensidades.

Limitados pela ausência de dados sobre \% $\mathrm{G}$ em lutadores de TKD de 10 a 14 anos, comparamos nossos valores com os de atletas ligeiramente mais velhos $(15,44 \pm 1,21$ anos), da seleção Junior de TKD dos USA (Pieter \& Falco, 2011). Pieter e Falco (2011) forneceram os valores das pregas adiposas destes atletas americanos e utilizamos estes dados para resolver a equação 4, o que resultou em uma média de $11,97 \%$ de gordura. Levando em conta o desvio padrão da nossa amostra, o \%G destes atletas americanos não parece ser significativamente menor que o atual. Esta semelhança fortalece a ideia de que nossa amostra possui $\% \mathrm{G}$ adequado a jovens atletas de "taekwondo".

A comparação de médias mostrou que a equação 4 subestimou o \%G em relação à DEXA, com EC e ET acima do sugerido por LOHMAN $(1992)(\leq 2,9 \% \mathrm{da}$ gordura relativa). Em relação aos resultados da regressão linear, a equação 4 apresentou um alto poder preditivo, de $87 \%$, com um EPE de 1\%, muito abaixo do valor máximo recomendado por LOHMAN (1992), que é de $3,5 \%$. Adicionalmente, a análise da concordância entre métodos (Análise de Bland e Altman) indicou que as diferenças de \%G entre a equação antropométrica utilizada e a DEXA apresentaram uma variabilidade interindividual aceitável (com amplitude dos limites de concordância inferior a 3,9\%). Ainda, nesta análise não houve enviesamento entre a diferença da gordura relativa dentre métodos e a média dentre estes. Isto significa que não houve tendência de subestimar o \%G em níveis de adiposidade mais reduzidos e superestimar o \%G em níveis de adiposidade mais elevados; ou de superestimar o \%G em níveis de adiposidade reduzida e subestimar o \%G em níveis de adiposidade elevada. Portanto, apesar de terem sido encontradas diferenças significativas entre os valores de $\% \mathrm{G}$ preditos e os obtidos pelo método de referência, a equação de Slaughter et al. (1988) demonstrou ser adequada para o universo restrito representado pela amostra desta pesquisa, desde que a equação 4 seja corrigida pela equação de ajuste $[\% \mathrm{G}(\mathrm{DEXA})=1,64+1,24$. $\% \mathrm{G}(\mathrm{Eq} 4)]$, resultante da regressão linear.

Um dos pontos fortes deste estudo prende-se com a reduzida publicação de estudos de validação de equações antropométricas em taekwondistas adolescentes. No entanto, é de assinalar como limitação o reduzido tamanho (n) da amostra. Este $\mathrm{n}$ reduzido se justifica devido à dificuldade de se encontrar atletas infantis de elite da modalidade em Portugal. Por outro lado, o critério de seleção por desempenho permitiu a obtenção de uma amostra bastante homogenia na variável analisada.

Em conclusão,os atletas da atual amostra obtiveram valores de $\% \mathrm{G}$ semelhantes aos de jovens lutadores americanos da seleção juniores, o que indicou que a composição corporal dos voluntários era adequada para o desempenho na modalidade.

Quanto à comparação de equações com o método de referência, apenas a equação 4 se mostrou adequada pelo cálculo amostral com cinco atletas. Embora esta equação tenha subestimado o $\% \mathrm{G}$ quanto ao método de referência, a mesma apresentou um alto poder explicativo, de $87 \%$, na predição dos valores obtidos pela DEXA; apresentou baixo EPE e baixos limites de concordância pela plotagem de Bland e ALTMAN (1986). Portanto, conclui-se que, desde que a equação de SLAUGHTER et al. (1988) seja corrigida pela equação de ajuste, esta é válida para a mensuração do \%G de taekwondistas adolescentes de elite de Portugal.

No entanto, as limitaçôes deste estudo permitem indicar que mais investigações devam ser realizadas, particularmente na validação de equaçôes para estimar alterações da \%G ao longo de uma época desportiva. 


\begin{abstract}
Analysis of predictive equations of body fat in young taekwondo athletes

Due to the lack of valid and accessible tests to measure the body fat percentage (BF\%) of adolescent taekwondo athletes (TKDA), this study aimed to analyze six anthropometric equations in the prediction of $\mathrm{BF} \%$ with the Dual-Energy-x-Ray (DEXA) as referential method for five TKDA (12.23 years \pm 1.60$)$. The BF\% estimated by the equations were compared with DEXA values using the t-student, linear regression and Bland \& Altman (B\&t A) tests. Only the equation of SlaUghter et al. (1988) was adequated by the sample size calculation, and although it subestimated the BF\% (in 4.85\% \pm 0.98 ), it showed a high correlation $\left(R=0.935 ; R^{2}=0.874 \quad E P E=1.01\right)$ and low amplitude in the limits of agreement at $95 \%(3.84 \%)$ by BCtA in comparison to DEXA. However, this equation is adequated to predict the BF\% in TKDA, if it's corrected by the adjustement equation [\%G (DEXA) $=1,64+1,24 \cdot \% \mathrm{G}$ (Eq 4)] generated by linear regression.
\end{abstract}

UnITERMS: Densitometry; Body composition; Skinfold-thickness.

\title{
Resumen
}

Análisis de ecuaciones predictivas de la grasa corporal en atletas jóvenes de taekwondo

Debido a la falta de métodos accesibles válidos para medir el porcentaje de grasa del cuerpo $(\% \mathrm{G})$ de atletas de taekwondo adolescentes (TKDA), destinada a analizar seis ecuaciones antropométricas de predicción de \%G, en cinco TKDA (12,23 años $\pm 1,60$ ), utilizando como método de referencia la Densitometria Radiológica de Energía Dual (DEXA). El \%G estimado por las ecuaciones fue comparado mediante la prueba t-student, regresión lineal y Bland \& Altman (BCtA) con los que se obtienen mediante DEXA. Sólo la ecuación del SLAUGHTER et al. (1988) fue adecuada para el cálculo del tamaño de la muestra y, a pesar de que había subestimado la $\% \mathrm{G}(4,85 \% \pm 0,98)$, este mostró una alta correlación $(R=0,935$; $\mathrm{R} 2=0,874 \mathrm{EPE}=1,01)$ y baja amplitud dentro de los límites de concordancia con el 95\% (3,84 \%) por B\&tA en comparación con la DEXA. Por lo tanto, esta ecuación demostró ser adecuada para la predicción de $\% \mathrm{G}$ en TKDA, desde que corrigida mediante la siguiente ecuación de ajuste [ $\% \mathrm{G}(\mathrm{DEXA})=1,64+$ $1,24 \cdot \% \mathrm{G}$ (eq 4)] generada mediante regresión lineal.

Palabras clave: Densitometría; Composición corporal; Pliegues cutáneos.

\section{Referências}

BLAND, J.M.; ALTMAN, D.G. Statistical methods for assessing agreement between two methods of clinical measurement. Lancet, London, v.1, n.8476, p.307-10, 1986.

BOILEAU, R.A.; LOHMAN, T.G.; SLAUGHTER, M.H. Exercise and body composition in children and youth. Scandinavian Journal of Sports Sciences, Copenhagen, v.7, p.17-27, 1985.

CAMPANOZZI, A.; DABBAS, M.; RUIZ, J.C.; RICOUR, C.; GOULET, O. Evaluation of lean body mass in obese children. European Journal of Pediatrics, Berlin, v.167, p.533-40, 2008.

COTUGNA, N.; VICKERY, C.E.; McBEE, S. Sports nutrition for young athletes. The Journal of School Nursing, Scarborough, v.21, p.323-8, 2005.

FLEMING, S.; COSTARELLI, V. Nutrient intake and body composition in relation to making weight in young male Taekwondo players. Nutrition \& Food Science, Bingley, v.37, p.358-66, 2007. 
GOBBO, L.A.; CYRINO, E.L.; PETROSKI, E.L.; CARDOS, J.R.; CARVALHO, F.O.; ROMANZINI, M.; AVELAR, A. Validação de equações antropométricas para a estimativa da massa muscular por meio de absormetria radiológica de dupla energia em universitários do sexo masculino. Revista Brasileira de Medicina do Esporte, São Paulo, v.14, p. 376-80, 2008. HELlER, J.; PERIC, T.; DLOUHÁ, R.; KOHLÍKOVÁ, E.; MELICHNA, J.; NOVÁKOVÁ, H. Physiological profiles of male and female taekwondo (ITF) black belts. Journal of Sports Sciences, London, v.16, p.243-9, 1998.

JOHNSTON, J.L.; LEONG, M.S.; CHECKLAND, E.G.; ZUBERBAHLER, P.C.; CONGER, P.R.; QUINNEY, H.A. Body fat assessed from body density and estimated from skinfold thickness in normal children and children with cystic fibrosis. The American Journal of Clinical Nutrition, Bethesda, v.48, p.1362-6, 1988.

LOFTIN, M.; NICHOLS, J.; GOING, S.; SOTHERN, M.; SCHMITZ, K.H.; RING, K.; STEVENS, J. Comparison of the validity of anthropometric and bioelectric impedance equations to assess body composition in adolescent girls. International Journal of Body Composition Research, London, v.5, p.1-8, 2007.

LOHMAN, T.G. Advances in body composition assessment. Champaign: Human Kinetics, 1992.

Applicability of body composition techniques and constants for children and youths. Exercise and Sports Sciences Reviews, Baltimore, v.14, p.325-7, 1986.

MUKHERJEE, D.; ROCHE, A.F. The estimation of percent body fat, body density and total body fat by maximum R2 regression equations. Human Biology, Detroit, v.56, p.79-109, 1984.

OLDS, T.; KANG, S.J. Anthropometric characteristics of adult male Korean taekwondo players. In: OLYMPIC TAEKWONDO ORGANIZING COMMITTEE (Ed.). Taekwondo and the new millennium-proceedings. Seoul: Korean National University of Physical Education, 2000. p.69-75.

PACCINI, M.K.; ARSA, G.; GLANER, M.F. Indicadores de gordura abdominal: antropometria vs absortometria de raio-x de dupla energia. Revista Brasileira de Cineantropometria e Desempenho Humano, Florianópolis, v. 10, p.283-8, 2008. PARIZKOVA, J. Total body fat and skinfold thickness in children. Metabolism: Clinical and Experimental, New York, v.10, p.794-809, 1961. PARKER, L.; REILLY, J.J.; SLATER, C.; WELLS, J.C.K.; PITSILADIS, Y. Validity of six field and laboratory methods for measurement of body composition in boys. Obesity Research, Baton Rouge, v.11, p.852-8, 2003.

PETRIE, H.J.; STOVER, E.A.; HORSWILL, C.A. Nutritional concerns for the child and adolescent competitor. Nutrition, Burbank, v.20, p.620-31, 2004.

PIETER, W.; BERCADES, L.T. Somatotypes of national elite combative sport athletes. Brazilian Journal of Biomotricity, Rio de Janeiro, v.3, p.21-30, 2009.

PIETER, W.; FALCO, C. Skinfold patering in elite Spanish and American junior taekwondo-in. Journal of Martial Arts Anthropology, Rzeszów, v.11, p.47-51, 2011.

QUITÉRIO, A.L.; SILVA, A.M.; CARNERO, E.A.; SARDINHA, L.B. Weekly training hours on body composition in young athetes. In: ACSM's ANNUAL MEETING, 54., New Orleans, 2007. Abstracts.... New Orleans: ACSM, 2007 RECH, C.R.; LIMA, L.R.A.; CORDEIRO, B.A.; PETROSKI, E.L.; VASCONCELOS, F.A.G. Validade de equações antropométricas para a estimativa da gordura corporal em idosos do sul do Brasil. Revista Brasileira de Cineantropometria e Desempenho Humano, Florianópolis, v.12, p.1-7, 2010.

RODRÍGUEZ, G.; MORENO, L.A.; BLAY, M.G.; BLAY, V.A.; FLETA, J.; SARRÍA, A.; BUENO, M. Body fat measurement in adolescents: comparison of skinfold thickness equation with dual-energy x-ray absormetry. European Journal of Clinical Nutrition, London, v.59, p.1158-66, 2005.

ROGOL, A.D.; CLARK, P.A.; ROEMMICH, J.N. Growth and pubertal development in children and adolescents: effects of diet and physical activity. American Journal of Clinical Nutrition, Bethesda, v.72, p.521-8, 2000.

SILVA, A.M.; FIELDS, D.A.; QUITÉRIO, A.L.; SARDINHA, L.B. Are skinfold-based models accurate and suitable for assessing changes in body composition in highly trained athletes? Journal of Strength and Conditioning Research, Champaign, v.23, p.1688-96, 2009.

SLAUGHTER, M.H.; LOHMAN, T.G.; BOILEAU, R.A.; HORSWILL, C.A.; STILLMAN, R.J.; VAN LOAN, M.D.; BEMBEN, D.A. Skinfold equations for estimation of body fatness in children and youths. Human Biology, Detroit, v.60, p.709-23, 1988. TAAFFE, D.; PIETER, W. Physical and physiological characteristic of elite taekwondo athlete, In: COMMONWEALTH AND INTERNATIONAL CONFERENCE ON PHYSICAL EDUCATION, SPORT, HEALTH, DANCE, RECREATION AND LEISURE, Auckland, 1990. Proceedings... Auckland: NZAHPER, 1990. v.3: Sport science Part 1, p.80-8. TANNER, J.M.; HEALY, M.J.R.; GOLDSTEIN, H.; CAMERON, N. Assessment of skeletal maturity and prediction of adult height [TW3 Method]. London: Harcourt, 2001.

TIMPSON, N.J.; SAYERS, A.; DAVEY-SMITH, G.; TOBIAS, J.H. How does body fat influence bone mass in childhood? a mendelian randomized approach. Journal of Bone And Mineral Research, New York, v.24, p.522-33, 2009. 
WANG, J.; THORTON, J.C.; KOLESNIK, S.; PIERSON, R.N. Anthropometry in body composition: an overview. Annals of the New York Academy of Sciences, New York, v.904, p.317-26, 2000.

WESTRATE, J.; DEURENBERG, P. Body composition in children: proposal for a method for calculating body fat percentage from total body density or skinfold thickness measurements. American Journal of Clinical Nutrition, Bethesda, v.50, p.1104-15, 1989 .

\begin{tabular}{c|l} 
& \\
ENDEREÇO & \\
Ret Moreira & Recebido para publicação: 30/ 10/2011 \\
$\begin{array}{c}\text { - BRto. } 441 \\
\text { - Revisado: } 21 / 05 / 2012 \\
\end{array}$ & Aceito: 25/05/2012 \\
&
\end{tabular}

